11-1-2009

\title{
Estimating the Parameters of Rayleigh Cumulative Exposure Model in Simple Step-Stress Testing. Natasha Beretvas is an
}

Mohammed Al-Haj Ebrahem

Yarmouk Universit, Irbid, Jordan, m_hassanb@hotmail.com

Abedel-Qader Al-Masri

YarmoukUniversit, Irbid, Jordan, almasri68@yahoo.com

Follow this and additional works at: http://digitalcommons.wayne.edu/jmasm

Part of the Applied Statistics Commons, Social and Behavioral Sciences Commons, and the Statistical Theory Commons

\section{Recommended Citation}

Ebrahem, Mohammed Al-Haj and Al-Masri, Abedel-Qader (2009) "Estimating the Parameters of Rayleigh Cumulative Exposure Model in Simple Step-Stress Testing. Natasha Beretvas is an," Journal of Modern Applied Statistical Methods: Vol. 8 : Iss. 2 , Article 12. DOI: $10.22237 /$ jmasm/ 1257034260

Available at: http://digitalcommons.wayne.edu/jmasm/vol8/iss2/12 


\title{
Estimating the Parameters of Rayleigh Cumulative Exposure Model in Simple Step-Stress Testing
}

\author{
Mohammed Al-Haj Ebrahem Abedel-Qader Al-Masri \\ Yarmouk University \\ Irbid, Jordan
}

Assumes the life distribution of a test unit for any stress follows a Rayleigh distribution with scale parameter $\theta$, and that $\operatorname{Ln}(\theta)$ is a linear function of the stress level. Maximum likelihood estimators of the parameters under a cumulative exposure model are obtained. The approximate variance estimates obtained from the asymptotic normal distribution of the maximum likelihood estimators are used to construct confidence intervals for the model parameters. A simulation study was conducted to study the performance of the estimators. Simulation results showed that in terms of bias, mean squared error, attainment of the nominal confidence level, symmetry of lower and upper error rates and the expected interval width, the estimators are very accurate and have a high level of precision.

Key words: Accelerated life test, Cumulative exposure model, Rayleigh distribution, Maximum likelihood estimation, Step-stress.

\section{Introduction}

The Rayleigh distribution arises in a variety of fields. This distribution is frequently employed by engineers and scientists as a model for data resulting from investigations involving wave propagation, radiation and related inquiries as well as in the analysis of target error data Cohen and Whitten (1988). Some types of electro vacuum devices age rapidly with time even though they may have no manufacturing defects, the Rayleigh distribution is quite appropriate for modeling the lifetime of such units as it possesses a linearly increasing hazard rate Johnson, Kotz and Balakrishnan (1994). Other applications and motivations for the Rayleigh distribution can be found in Cohen and Whitten (1988).

Mohammed Al-Haj Ebrahem is an Associate Professor in the Department of Statistics. Email: m_hassanb@hotmail.com. Abedel-Qader AlMasri is an Instructor in the Department of Statistics. Email: almasri68@yahoo.com
Accelerated life tests are used to quickly obtain information on the life distribution of products by testing them at higher than nominal levels of stress to induce early failures. Data are obtained at accelerated conditions and based on a regression type model, results are extrapolated to the design stress to estimate the life distribution; such overstress testing reduces time and cost. One method of applying stress to the test units is a step-stress scheme which allows the stress of a unit to be changed at specified times. Nelson (1980) described this important type of accelerated life test. In step-stress testing, a unit is placed on a test at an initial low stress, if it does not fail in a predetermined time, $\tau$, stress is increased. If there is a single change of stress, the accelerated life test is called a simple step-stress test.

The cumulative exposure model defined by Nelson (1990) for simple step-stress testing with low stress $X_{1}$ and high stress $X_{2}$ is:

$$
G(t)=\left\{\begin{array}{cl}
G_{1}(t) & t \leq \tau \\
G_{2}(t-\tau+s) & t>\tau
\end{array}\right.
$$

where $G_{i}(t)$ is the cumulative distribution function of the failure time at stress $X_{i}, \tau$ is the 


\section{AL-HAJ EBRAHEM \& AL-MASRI}

time to change stress and $S$ is the solution of $G_{1}(\tau)=G_{2}(s)$.

Most of the available literature on a step-stress accelerated life testing deals with the exponential exposure model. Khamis and Higgins $(1996,1998)$ proposed a new model known as $\mathrm{KH}$ model for step-stress accelerated life test as an alternative to the Weibull cumulative exposure model.

Miller and Nelson (1983) obtained the optimum simple step-stress accelerated life test plans for the case where the test units have exponentially distributed lifetimes. Bai, Kim and Lee (1989) extended the results of Miller and Nelson (1983) to the case of censoring. Khamis and Higgins (1996) obtained the optimum 3-step step-stress using the exponential distribution. Alhadeed and Yang (2005) obtained the optimum design for the lognormal step-stress model. Al-Haj Ebrahem and Al Masri (2007(a)) obtained the optimum simple step-stress plans for the log-logistic cumulative exposure model, by minimizing the asymptotic variance of the maximum likelihood estimate of a given $100 \mathrm{P}$ th percentile of the distribution at the design stress.

Al-Haj Ebrahem and Al Masri (2007(b)) obtained the optimum simple step-stress plans for the log-logistic distribution under time censoring. Xiong (1998) presented the inferences of parameters in the simple stepstress model in accelerated life testing with type two censoring. Xiong and Milliken (2002) studied statistical models in step-stress accelerated life testing when stress change time are random and obtained the marginal life distribution for test units. Nonparametric approaches for step-stress testing have been proposed by Shaked and Singurwalla (1983) and Schmoyer (1991). For additional details, see Chung and Bai (1998) and Gouno (2001). This article considers point and interval estimation of Rayleigh cumulative exposure model parameters.

\section{Model and Assumptions}

The probability density function and the cumulative distribution function of the Rayleigh distribution are given respectively by:

$$
f(y, \theta)=\left\{\begin{array}{cl}
\frac{2 y}{\theta} e^{\frac{-y^{2}}{\theta}} & , y>0, \theta>0 \\
0 & , \text { otherwise }
\end{array}\right.
$$

and

$$
F(y, \theta)=\left\{\begin{array}{cl}
0 & , y<0 \\
1-e^{\frac{-y^{2}}{\theta}} & , y>0
\end{array}\right.
$$

The following assumptions are understood:

1. Under any stress the lifetime of a test unit follows a Rayleigh distribution.

2. Testing is conducted at stresses $X_{1}$ and $X_{2}$, where $\mathrm{X}_{1}<\mathrm{X}_{2}$.

3. The relationship between the parameter $\theta_{i}$ and the stress $X_{i}$ is given by $\operatorname{Ln}\left(\theta_{i}\right)=\beta_{0}+\beta_{1} X_{i}$, where $\beta_{0}$ and $\beta_{1}$ are unknown parameters to be determined from the test data.

4. The lifetimes of test units are independent and identically distributed.

5. All $\mathrm{n}$ units are initially placed on low stress $\mathrm{X}_{1}$ and run until time $\tau$ when the stress is changed to high stress $\mathrm{X}_{2}$. At $\mathrm{X}_{2}$ testing continues until all remaining units fail.

Verification that the Rayleigh cumulative exposure model for step-stress is given by:

$$
G(y)=\left\{\begin{array}{cl}
1-e^{\frac{-y^{2}}{\theta_{1}}} & , 0<y<\tau \\
\left.1-e^{\left.\frac{-(y-\tau+\tau}{\theta_{2}}\right)^{\theta_{2}}}\right)^{2} & , \tau<y<\infty
\end{array}\right.
$$

If $T=Y^{2}$, then the cumulative exposure model of $\mathrm{T}$ is given by:

$$
G(t)=\left\{\begin{array}{cl}
1-e^{\frac{-t}{\theta_{1}}} & , 0<t<\tau^{2} \\
1-e^{\left.\frac{-(\sqrt{t}-\tau+\tau}{\frac{\theta_{2}}{\theta_{1}}}\right)^{2}} & , \tau^{2}<t<\infty
\end{array}\right.
$$




\section{RAYLEIGH MODEL PARAMETER ESTIMATION IN STEP-STRESS TESTING}

Note that $G(t)$ is not a step-stress exponential cumulative exposure model. For simplicity, let $\tau_{1}=\tau^{2}$, so that the cumulative exposure model of $\mathrm{T}$ is given by:

$$
G(t)=\left\{\begin{array}{cc}
1-e^{\frac{-t}{\theta_{1}}} & , 0<t<\tau_{1} \\
1-e^{\frac{-\left(\sqrt{t}-\sqrt{\tau_{1}}+\sqrt{\frac{\tau_{1} \theta_{2}}{\theta_{1}}}\right)^{2}}{\theta_{2}}} & , \tau_{1}<t<\infty
\end{array}\right.
$$

and the corresponding probability density function of $\mathrm{T}$ is given by:

$$
\begin{aligned}
& g(t)= \\
& \left\{\begin{array}{cl}
\frac{1}{\boldsymbol{\theta}_{1}} e^{\frac{-t}{\boldsymbol{\theta}_{1}}} & , 0<t<\boldsymbol{\tau}_{1} \\
\frac{\left(\sqrt{t}-\sqrt{\boldsymbol{\tau}_{1}}+\sqrt{\frac{\boldsymbol{\tau}_{1} \boldsymbol{\theta}_{2}}{\boldsymbol{\theta}_{1}}}\right)}{\boldsymbol{\theta}_{2} \sqrt{t}} e^{\frac{-\left(\sqrt{t}-\sqrt{\tau_{1}}+\sqrt{\frac{\tau_{\boldsymbol{\theta}_{2}}}{\boldsymbol{\theta}_{1}}}\right)^{2}}{\boldsymbol{\theta}_{2}}} & , \tau_{1}<t<\infty
\end{array}\right.
\end{aligned}
$$

\section{Methodology}

Model Parameters Estimation

Let $t_{i j}, j=1,2, \ldots, n_{i}, i=1,2$ be the observed lifetime under low and high stress, where $n_{1}$ denotes the number of units failed at the low stress $\mathrm{X}_{1}$ and $n_{2}$ denotes the number of units failed at the high stress $\mathrm{X}_{2}$. The Likelihood function is given by:

$$
\begin{aligned}
& L\left(t_{i j}, \boldsymbol{\beta}_{0}, \boldsymbol{\beta}_{1}\right)= \\
& \boldsymbol{\theta}_{1}^{-n_{1}} \boldsymbol{\theta}_{2}^{-n 2} e^{-\frac{\sum_{j=1}^{n_{1}} t_{1 j}}{\boldsymbol{\theta}_{1}}-\frac{\sum_{j=1}^{n_{2}}\left(\sqrt{t_{2 j}}-\sqrt{\tau_{1}}+\sqrt{\frac{\boldsymbol{\tau}_{1} \boldsymbol{\theta}_{2}}{\boldsymbol{\theta}_{1}}}\right)^{2}}{\boldsymbol{\theta}_{2}}} \\
& * \prod_{j=1}^{n_{2}}\left(1-\sqrt{\frac{\boldsymbol{\tau}_{1}}{t_{2 j}}}+\sqrt{\frac{\boldsymbol{\tau}_{1} \boldsymbol{\theta}_{2}}{t_{2 j} \boldsymbol{\theta}_{1}}}\right)
\end{aligned}
$$

$$
\boldsymbol{\theta}_{1}=e^{\boldsymbol{\beta}_{0}+\boldsymbol{\beta}_{1} X_{1}} \text { and } \boldsymbol{\theta}_{2}=e^{\boldsymbol{\beta}_{0}+\boldsymbol{\beta}_{1} X_{2}} \text {. }
$$

The log likelihood function is given by:

$$
\begin{aligned}
\operatorname{LnL}\left(t_{i j}, \boldsymbol{\beta}_{0}, \boldsymbol{\beta}_{1}\right)= & \\
& -n_{1} \ln \left(\boldsymbol{\theta}_{1}\right)-n_{2} \ln \left(\boldsymbol{\theta}_{2}\right) \\
& +\sum_{j=1}^{n_{2}} \operatorname{Ln}\left(1-\sqrt{\frac{\boldsymbol{\tau}_{1}}{t_{2 j}}}+\sqrt{\frac{\boldsymbol{\tau}_{1} \boldsymbol{\theta}_{2}}{t_{2 j} \boldsymbol{\theta}_{1}}}\right) \\
& -\frac{\sum_{j=1}^{n_{1}} t_{1 j}}{\boldsymbol{\theta}_{1}}-\frac{\sum_{j=1}^{n_{2}}\left(\sqrt{t_{2 j}}-\sqrt{\tau_{1}}+\sqrt{\frac{\tau_{1} \boldsymbol{\theta}_{2}}{\boldsymbol{\theta}_{1}}}\right)^{2}}{\boldsymbol{\theta}_{2}}
\end{aligned}
$$

The maximum likelihood estimates $\hat{\beta}_{0}$ and $\hat{\beta}_{1}$ for the model parameters $\beta_{0}$ and $\beta_{1}$ can be obtained by solving numerically the following two equations:

$$
\begin{aligned}
& \frac{\partial \operatorname{LnL}\left(t_{i j}, \beta_{0}, \beta_{1}\right)}{\partial \beta_{0}}=0 \\
& \frac{\partial \operatorname{Ln} L\left(t_{i j}, \beta_{0}, \beta_{1}\right)}{\partial \beta_{1}}=0
\end{aligned}
$$

In order to construct confidence intervals for the model parameters, the asymptotic normality of the maximum likelihood estimates are used. It is known that:

$$
\left(\hat{\beta}_{0}, \hat{\beta}_{1}\right) \sim N\left(\left(\beta_{0}, \beta_{1}\right), \hat{F}^{-1}\right)
$$

where $\hat{F}^{-1}$ denotes the inverse of the observed Fisher information matrix $\hat{F}$. The observed Fisher information matrix $\hat{F}$ is obtained by evaluating the second and mixed partial derivatives of $\operatorname{LnL}\left(t_{i j}, \beta_{0}, \beta_{1}\right)$ at the maximum likelihood estimates $\hat{\beta}_{0}$ and $\hat{\beta}_{1}$, that is:

$$
\hat{F}=\left[\begin{array}{ll}
\hat{F}_{11} & \hat{F}_{12} \\
\hat{F}_{21} & \hat{F}_{22}
\end{array}\right]
$$




\section{AL-HAJ EBRAHEM \& AL-MASRI}

where

$$
\begin{gathered}
\hat{F}_{11}=-\left.\frac{\partial^{2} \operatorname{LnL}\left(t_{i j}, \beta_{0}, \beta_{1}\right)}{\partial \beta_{0}^{2}}\right|_{\beta_{0}=\hat{\beta}_{0}, \beta_{1}=\hat{\beta}_{1}} \\
\hat{F}_{12}=\hat{F}_{21}=-\left.\frac{\partial^{2} \operatorname{LnL}\left(t_{i j}, \beta_{0}, \beta_{1}\right)}{\partial \beta_{0} \partial \beta_{1}}\right|_{\beta_{0}=\hat{\beta}_{0}, \beta_{1}=\hat{\beta}_{1}} \\
\hat{F}_{22}=-\left.\frac{\partial^{2} \operatorname{LnL}\left(t_{i j}, \beta_{0}, \beta_{1}\right)}{\partial \beta_{1}^{2}}\right|_{\beta_{0}=\hat{\beta}_{0}, \beta_{1}=\hat{\beta}_{1}}
\end{gathered}
$$

Thus, a $(1-\alpha) 100 \%$ confidence interval for the model parameter $\beta_{l}, l=0,1$ is given by:

$$
\left[\hat{\boldsymbol{\beta}}_{l}-Z_{1-\boldsymbol{\alpha} / 2} S . E\left(\hat{\boldsymbol{\beta}}_{l}\right), \quad \hat{\boldsymbol{\beta}}_{l}+Z_{1-\boldsymbol{\alpha} / 2} S . E\left(\hat{\boldsymbol{\beta}}_{l}\right)\right]
$$

where $S . E\left(\hat{\beta}_{l}\right)$ denotes the standard error of the maximum likelihood estimates $\hat{\beta}_{l}$ which is the square root of the diagonal element of $\hat{F}^{-1}$, and $Z_{1-\alpha / 2}$ is the $(1-\alpha / 2)$ percentile of the standard normal distribution.

Note that an optimal test plan can be determined by minimizing with respect to the change time $\tau_{1}$ the asymptotic variance at the design stress $\mathrm{X}_{0}$. Thus, the numerical search method was used to find the value of $\tau_{1}^{*}$ that minimizes $\quad\left(1 X_{0}\right) \hat{F}^{-1}\left(1 X_{0}\right)^{T}$, where $\left(1 X_{0}\right)^{T}$ denotes the transpose of the vector (1 $X_{0}$ ). Thus the optimum time to change stress under the Rayleigh cumulative exposure model is $\tau^{*}=\sqrt{\tau_{1}^{*}}$.

\section{Example}

The data in Table 1 includes $\mathrm{n}=\left(\mathrm{n}_{1}+\mathrm{n}_{2}\right)$ $=30$ simulated observations from cumulative exposure model (5) defined above. The values used in this example are: $\beta_{0}=2, \beta_{1}=4, \tau_{1}^{*}=$ 28.4 .
Table 1: Simulated Data

\begin{tabular}{|c|ccc|}
\hline Stress & \multicolumn{3}{|c|}{ Failure Times } \\
\hline \multirow{5}{*}{$\mathrm{X}_{1}=0.2$} & 4.49977 & 7.17595 & 13.8035 \\
& 20.4499 & 1.89708 & 12.0347 \\
& 20.8349 & 23.9302 & 7.48286 \\
& 4.14598 & 0.101846 & 0.535875 \\
& 7.20451 & 12.7104 & 14.0179 \\
& 13.2136 & 23.3564 & 26.5207 \\
& 8.30107 & 1.3215 & \\
\hline \multirow{5}{*}{$\mathrm{X}_{2}=1$} & 262.761 & 645.625 \\
& 152.777 & 81.7587 \\
& 589.63 & 65.7081 \\
& 575.368 & 100.604 \\
\hline
\end{tabular}

The simulated data results show:

1. The values of the maximum likelihood estimates are $\hat{\beta}_{0}=2.45473, \hat{\beta}_{1}=4.10729$.

2. The inverse of the observed Fisher information matrix is $\hat{F}^{-1}=\left[\begin{array}{cc}0.119688 & -0.235227 \\ -0.235227 & 0.640747\end{array}\right]$

3. A $95 \%$ confidence interval for $\beta_{0}$ is [1.77665, 3.13281].

4. A $95 \%$ confidence interval for $\beta_{1}$ is [2.53838, 5.6762].

Simulation Study

A simulation study was conducted to investigate the performance of the maximum likelihood estimates, and the performance of the confidence interval based on the asymptotic normality of the maximum likelihood estimates. The criteria used for the evaluation of the performance of the maximum likelihood estimates were the bias and the mean squared error (MSE). For the confidence interval with confidence coefficient $(1-\alpha)$ the following were calculated:

1. The expected width (W): the average width of the simulated intervals.

2. Lower error rate (L): the fraction of intervals that fall entirely above the true parameter. 


\section{RAYLEIGH MODEL PARAMETER ESTIMATION IN STEP-STRESS TESTING}

3. Upper error rate $(U)$ : the fraction of intervals that fall entirely below the true parameters.

4. Total error rate $(T)$ : the fraction of intervals that did not contain the true parameter value.

The indices of the simulation study were:

- $\mathrm{n}$ : total number of units placed on the test, $\mathrm{n}=10,40,80,100$.

- $\mathrm{X}_{1}$ : low stress level, $\mathrm{X}_{1}=0.1,0.2,0.3$, 0.5 .

- $\mathrm{X}_{2}$ : high stress level, $\mathrm{X}_{2}=0.9,1.0,1.2$, 1.3, 1.9 .

- For $\beta_{0}=4, \beta_{1}=6, \alpha=0.05$ and for each combination of $n, X_{1}$ and $X_{2} 2,000$ samples were generated.

Results

Tables 2-5 show simulation results for parameter $\beta_{0}$, while Tables 6-9 show simulation results for parameter $\beta_{1}$.

\section{Conclusion}

Based on the simulation results the following conclusions are put forth. For the parameter $\beta_{0}$, the maximum likelihood estimate $\hat{\beta}_{0}$ has small values of bias and mean squared error, also as the sample size increases the value of the bias and the mean squared error decreases. The confidence interval for $\hat{\beta}_{0}$ had a small expected width value and the expected width decreases as the sample size increases. In terms of attainment of the coverage probability and the symmetry of lower and upper error rates, the intervals behave very well especially for large value of $n$. Also, from the results it appears that, for the same value of $X_{2}$, as the value of $X_{1}$ increases the values of expected width, bias and mean squared error also increase. Conversely, for the same value of $\mathrm{X}_{1}$, as the value of $\mathrm{X}_{2}$ increases the values of expected width, bias and mean squared error decrease. Thus, the recommendation is to use a small value of $X_{1}$ and a large value of $X_{2}$, and the same conclusions can be drawn for the parameter $\beta_{1}$.

Table 2: Simulation Results of the Parameter $\beta_{0}$ when $\mathrm{n}=10$

\begin{tabular}{|c|c|c|c|c|c|c|c|}
\hline $\mathrm{X}_{2}$ & $\mathrm{X}_{1}$ & $\mathrm{~W}$ & $\mathrm{~L}$ & $\mathrm{U}$ & $\mathrm{T}$ & Bias & MSE \\
\hline \multirow{4}{*}{0.9} & 0.1 & 2.1703 & 0.0440 & 0.0000 & 0.0440 & 0.2576 & 0.2089 \\
\cline { 2 - 8 } & 0.2 & 3.0980 & 0.0415 & 0.0000 & 0.0415 & 0.2607 & 0.4146 \\
\cline { 2 - 8 } & 0.3 & 4.1653 & 0.0505 & 0.0015 & 0.0520 & 0.3094 & 0.8260 \\
\cline { 2 - 8 } & 0.5 & 7.9925 & 0.0540 & 0.0040 & 0.0580 & 0.5476 & 3.1766 \\
\hline \multirow{4}{*}{1.0} & 0.1 & 1.9623 & 0.0380 & 0.0005 & 0.0385 & 0.2499 & 0.1951 \\
\cline { 2 - 8 } & 0.2 & 3.0753 & 0.0390 & 0.0005 & 0.0395 & 0.2477 & 0.3675 \\
\cline { 2 - 8 } & 0.3 & 3.8550 & 0.0525 & 0.0025 & 0.0550 & 0.3097 & 0.7143 \\
\cline { 2 - 8 } & 0.5 & 6.0328 & 0.0435 & 0.0050 & 0.0485 & 0.4283 & 2.1923 \\
\hline \multirow{4}{*}{1.3} & 0.1 & 1.9296 & 0.0430 & 0.0000 & 0.0430 & 0.2543 & 0.1796 \\
\cline { 2 - 8 } & 0.2 & 2.6484 & 0.0410 & 0.0010 & 0.0420 & 0.2520 & 0.2965 \\
\cline { 2 - 8 } & 0.3 & 3.3296 & 0.0520 & 0.0010 & 0.0530 & 0.3024 & 0.5133 \\
\cline { 2 - 8 } & 0.5 & 4.9964 & 0.0540 & 0.0025 & 0.0565 & 0.3945 & 1.3313 \\
\cline { 2 - 8 } & 0.1 & 1.8004 & 0.0350 & 0.0005 & 0.0355 & 0.2496 & 0.1637 \\
\cline { 2 - 8 } & 0.2 & 2.4806 & 0.0435 & 0.0010 & 0.0445 & 0.2625 & 0.2787 \\
\cline { 2 - 8 } & 0.3 & 3.1185 & 0.0395 & 0.0030 & 0.0425 & 0.2600 & 0.4204 \\
\hline \multirow{5}{*}{1.9} & 0.5 & 4.6224 & 0.0445 & 0.0025 & 0.0470 & 0.3078 & 1.1109 \\
\cline { 2 - 8 } & 0.1 & 1.6907 & 0.0460 & 0.0005 & 0.0465 & 0.2815 & 0.1631 \\
\cline { 2 - 8 } & 0.2 & 2.1678 & 0.0390 & 0.0010 & 0.0400 & 0.2471 & 0.1990 \\
\cline { 2 - 8 } & 0.3 & 2.5498 & 0.0325 & 0.0010 & 0.0335 & 0.2292 & 0.2569 \\
\hline & 0.5 & 3.5230 & 0.0465 & 0.0015 & 0.0480 & 0.2602 & 0.5334 \\
\hline
\end{tabular}




\section{AL-HAJ EBRAHEM \& AL-MASRI}

Table 3: Simulation Results of the Parameter $\beta_{0}$ when $\mathrm{n}=40$

\begin{tabular}{|c|c|c|c|c|c|c|c|}
\hline $\mathrm{X}_{2}$ & $\mathrm{X}_{1}$ & $\mathrm{~W}$ & $\mathrm{~L}$ & $\mathrm{U}$ & $\mathrm{T}$ & Bias & MSE \\
\hline \multirow{4}{*}{0.9} & 0.1 & 0.9862 & 0.0375 & 0.0090 & 0.0465 & 0.0439 & 0.0563 \\
\cline { 2 - 8 } & 0.2 & 1.2743 & 0.0425 & 0.0125 & 0.0550 & 0.0447 & 0.1110 \\
\cline { 2 - 8 } & 0.3 & 1.7032 & 0.0415 & 0.0100 & 0.0515 & 0.0609 & 0.1921 \\
\cline { 2 - 8 } & 0.5 & 3.1698 & 0.0390 & 0.0155 & 0.0545 & 0.1073 & 0.6790 \\
\hline \multirow{4}{*}{1.0} & 0.1 & 1.0098 & 0.0345 & 0.0105 & 0.0450 & 0.0491 & 0.0511 \\
\cline { 2 - 8 } & 0.2 & 1.2215 & 0.0460 & 0.0100 & 0.0560 & 0.0585 & 0.0969 \\
\cline { 2 - 8 } & 0.3 & 1.5599 & 0.0390 & 0.0125 & 0.0515 & 0.0621 & 0.1616 \\
\cline { 2 - 8 } & 0.5 & 2.6856 & 0.0355 & 0.0140 & 0.0495 & 0.0758 & 0.4816 \\
\hline \multirow{4}{*}{1.2} & 0.1 & 0.9224 & 0.0360 & 0.0045 & 0.0405 & 0.0468 & 0.0450 \\
\cline { 2 - 8 } & 0.2 & 1.1081 & 0.0375 & 0.0105 & 0.0480 & 0.0368 & 0.0793 \\
\cline { 2 - 8 } & 0.3 & 1.3694 & 0.0350 & 0.0075 & 0.0425 & 0.0489 & 0.1197 \\
\hline & 0.5 & 2.1300 & 0.0385 & 0.0175 & 0.0560 & 0.0587 & 0.3000 \\
\cline { 2 - 8 } & 0.1 & 0.8921 & 0.0310 & 0.0100 & 0.0410 & 0.0360 & 0.0419 \\
\cline { 2 - 8 } & 0.2 & 1.0858 & 0.0325 & 0.0065 & 0.0390 & 0.0451 & 0.0686 \\
\cline { 2 - 8 } & 0.3 & 1.3059 & 0.0350 & 0.0135 & 0.0485 & 0.0508 & 0.1135 \\
\hline \multirow{4}{*}{1.9} & 0.5 & 1.9469 & 0.0360 & 0.0155 & 0.0515 & 0.0732 & 0.2551 \\
\cline { 2 - 8 } & 0.1 & 0.8267 & 0.0290 & 0.0070 & 0.0360 & 0.0476 & 0.0328 \\
\cline { 2 - 8 } & 0.2 & 1.0231 & 0.0270 & 0.0060 & 0.0330 & 0.0411 & 0.0512 \\
\cline { 2 - 7 } & 0.3 & 1.1547 & 0.0360 & 0.0130 & 0.0490 & 0.0443 & 0.0767 \\
\hline
\end{tabular}

Table 4: Simulation Results of the Parameter $\beta_{0}$ when $\mathrm{n}=80$

\begin{tabular}{|c|c|c|c|c|c|c|c|}
\hline $\mathrm{X}_{2}$ & $\mathrm{X}_{1}$ & $\mathrm{~W}$ & $\mathrm{~L}$ & $\mathrm{U}$ & $\mathrm{T}$ & Bias & MSE \\
\hline \multirow{4}{*}{0.9} & 0.1 & 0.6529 & 0.0345 & 0.0210 & 0.0555 & 0.0100 & 0.0276 \\
\cline { 2 - 8 } & 0.2 & 0.8844 & 0.0345 & 0.0170 & 0.0515 & 0.0305 & 0.0504 \\
\cline { 2 - 8 } & 0.3 & 1.1884 & 0.0380 & 0.0145 & 0.0525 & 0.0313 & 0.0932 \\
\cline { 2 - 8 } & 0.5 & 2.2149 & 0.0405 & 0.0160 & 0.0565 & 0.0706 & 0.3394 \\
\hline \multirow{4}{*}{1.0} & 0.1 & 0.6313 & 0.0295 & 0.0140 & 0.0435 & 0.0134 & 0.0234 \\
\cline { 2 - 8 } & 0.2 & 0.8338 & 0.0325 & 0.0105 & 0.0430 & 0.0314 & 0.0443 \\
\cline { 2 - 8 } & 0.3 & 1.0872 & 0.0355 & 0.0195 & 0.0550 & 0.0269 & 0.0809 \\
\cline { 2 - 8 } & 0.5 & 1.8748 & 0.0340 & 0.0145 & 0.0485 & 0.0577 & 0.2303 \\
\hline \multirow{4}{*}{1.3} & 0.1 & 0.6049 & 0.0330 & 0.0160 & 0.0490 & 0.0133 & 0.0238 \\
\cline { 2 - 8 } & 0.2 & 0.7612 & 0.0400 & 0.0130 & 0.0530 & 0.0254 & 0.0379 \\
\cline { 2 - 8 } & 0.3 & 0.9534 & 0.0350 & 0.0180 & 0.0530 & 0.0278 & 0.0596 \\
\cline { 2 - 8 } & 0.5 & 1.4828 & 0.0335 & 0.0125 & 0.0460 & 0.0403 & 0.1381 \\
\cline { 2 - 8 } & 0.1 & 0.5973 & 0.0250 & 0.0120 & 0.0370 & 0.0121 & 0.0220 \\
\cline { 2 - 8 } & 0.2 & 0.7351 & 0.0395 & 0.0180 & 0.0575 & 0.0274 & 0.0361 \\
\cline { 2 - 8 } & 0.3 & 0.9059 & 0.0320 & 0.0205 & 0.0525 & 0.0249 & 0.0560 \\
\hline \multirow{4}{*}{1.9} & 0.1 & 1.3628 & 0.0360 & 0.0210 & 0.0570 & 0.0324 & 0.1259 \\
\cline { 2 - 8 } & 0.2 & 0.5604 & 0.0250 & 0.0120 & 0.0370 & 0.0174 & 0.0173 \\
\cline { 2 - 7 } & 0.3 & 0.7435 & 0.0295 & 0.0165 & 0.0460 & 0.0161 & 0.0338 \\
\cline { 2 - 7 } & 0.5 & 0.9887 & 0.0310 & 0.0165 & 0.0475 & 0.0200 & 0.0631 \\
\hline
\end{tabular}


RAYLEIGH MODEL PARAMETER ESTIMATION IN STEP-STRESS TESTING

Table 5: Simulation Results of the Parameter $\beta_{0}$ when $\mathrm{n}=100$

\begin{tabular}{|c|c|c|c|c|c|c|c|}
\hline $\mathrm{X}_{2}$ & $\mathrm{X}_{1}$ & $\mathrm{~W}$ & $\mathrm{~L}$ & $\mathrm{U}$ & $\mathrm{T}$ & Bias & MSE \\
\hline \multirow{4}{*}{0.9} & 0.1 & 0.5793 & 0.0285 & 0.0150 & 0.0435 & 0.0140 & 0.0211 \\
\cline { 2 - 8 } & 0.2 & 0.7890 & 0.0320 & 0.0195 & 0.0515 & 0.0198 & 0.0414 \\
\cline { 2 - 8 } & 0.3 & 1.0585 & 0.0460 & 0.0170 & 0.0630 & 0.0309 & 0.0787 \\
\cline { 2 - 8 } & 0.5 & 1.9750 & 0.0290 & 0.0150 & 0.0440 & 0.0467 & 0.2473 \\
\hline \multirow{4}{*}{1.0} & 0.1 & 0.5612 & 0.0295 & 0.0095 & 0.0390 & 0.0178 & 0.0193 \\
\cline { 2 - 8 } & 0.2 & 0.7432 & 0.0305 & 0.0160 & 0.0465 & 0.0201 & 0.0354 \\
\cline { 2 - 8 } & 0.3 & 0.9699 & 0.0335 & 0.0175 & 0.0510 & 0.0295 & 0.0621 \\
\cline { 2 - 8 } & 0.5 & 1.6748 & 0.0325 & 0.0205 & 0.0530 & 0.0284 & 0.1858 \\
\hline \multirow{4}{*}{1.2} & 0.1 & 0.5354 & 0.0255 & 0.0125 & 0.0380 & 0.0141 & 0.0169 \\
\cline { 2 - 8 } & 0.2 & 0.6790 & 0.0355 & 0.0190 & 0.0545 & 0.0158 & 0.0313 \\
\cline { 2 - 8 } & 0.3 & 0.8494 & 0.0375 & 0.0150 & 0.0525 & 0.0192 & 0.0465 \\
\cline { 2 - 8 } & 0.5 & 1.3251 & 0.0325 & 0.0205 & 0.0530 & 0.0227 & 0.1167 \\
\cline { 2 - 8 } & 0.1 & 0.5261 & 0.0260 & 0.0150 & 0.0410 & 0.0172 & 0.0171 \\
\cline { 2 - 8 } & 0.2 & 0.6556 & 0.0250 & 0.0200 & 0.0450 & 0.0154 & 0.0264 \\
\cline { 2 - 8 } & 0.3 & 0.8070 & 0.0355 & 0.0160 & 0.0515 & 0.0225 & 0.0420 \\
\hline \multirow{4}{*}{1.9} & 0.5 & 1.2151 & 0.0290 & 0.0220 & 0.0510 & 0.0164 & 0.0977 \\
\cline { 2 - 8 } & 0.1 & 0.4892 & 0.0275 & 0.0170 & 0.0445 & 0.0151 & 0.0150 \\
\cline { 2 - 7 } & 0.2 & 0.5702 & 0.0280 & 0.0170 & 0.0450 & 0.0137 & 0.0205 \\
\cline { 2 - 7 } & 0.3 & 0.6626 & 0.0315 & 0.0195 & 0.0510 & 0.0167 & 0.0283 \\
\hline
\end{tabular}

Table 6: Simulation Results of the Parameter $\beta_{1}$ when $\mathrm{n}=10$

\begin{tabular}{|c|c|c|c|c|c|c|c|}
\hline $\mathrm{X}_{2}$ & $\mathrm{X}_{1}$ & $\mathrm{~W}$ & $\mathrm{~L}$ & $\mathrm{U}$ & $\mathrm{T}$ & $\mathrm{Bias}$ & $\mathrm{MSE}$ \\
\hline \multirow{4}{*}{0.9} & 0.1 & 11.7520 & 0.0000 & 0.1050 & 0.1050 & -1.1302 & 5.4630 \\
\cline { 2 - 8 } & 0.2 & 10.7495 & 0.0000 & 0.0920 & 0.0920 & -0.9623 & 5.0870 \\
\cline { 2 - 8 } & 0.3 & 10.6093 & 0.0005 & 0.0835 & 0.0840 & -0.8507 & 5.5120 \\
\cline { 2 - 8 } & 0.5 & 13.7981 & 0.0020 & 0.0765 & 0.0785 & -0.9891 & 9.4010 \\
\hline \multirow{4}{*}{1.0} & 0.1 & 9.5277 & 0.0000 & 0.1020 & 0.1020 & -0.9831 & 4.4160 \\
\cline { 2 - 8 } & 0.2 & 10.6398 & 0.0005 & 0.0980 & 0.0985 & -0.8012 & 4.0510 \\
\cline { 2 - 8 } & 0.3 & 9.6039 & 0.0005 & 0.0945 & 0.0950 & -0.8252 & 4.5300 \\
\cline { 2 - 8 } & 0.5 & 9.9503 & 0.0025 & 0.0700 & 0.0725 & -0.7903 & 6.2630 \\
\hline \multirow{4}{*}{1.2} & 0.1 & 9.0816 & 0.0000 & 0.1130 & 0.1130 & -0.8944 & 3.1220 \\
\cline { 2 - 8 } & 0.2 & 8.4417 & 0.0000 & 0.0945 & 0.0945 & -0.7595 & 2.8450 \\
\cline { 2 - 8 } & 0.3 & 7.8835 & 0.0015 & 0.0985 & 0.1000 & -0.7365 & 2.9810 \\
\cline { 2 - 8 } & 0.5 & 7.9438 & 0.0005 & 0.0795 & 0.0800 & -0.6901 & 3.5090 \\
\hline \multirow{4}{*}{1.3} & 0.1 & 7.6321 & 0.0000 & 0.0980 & 0.0980 & -0.7888 & 2.5240 \\
\cline { 2 - 8 } & 0.2 & 7.5746 & 0.0005 & 0.1070 & 0.1075 & -0.7344 & 2.5700 \\
\cline { 2 - 8 } & 0.3 & 7.1972 & 0.0000 & 0.0840 & 0.0840 & -0.6107 & 2.2610 \\
\cline { 2 - 8 } & 0.5 & 7.2356 & 0.0000 & 0.0755 & 0.0755 & -0.5331 & 2.8070 \\
\hline \multirow{4}{*}{1.9} & 0.1 & 6.0578 & 0.0000 & 0.1165 & 0.1165 & -0.6138 & 1.3170 \\
\cline { 2 - 8 } & 0.2 & 5.9001 & 0.0000 & 0.1085 & 0.1085 & -0.5047 & 1.1770 \\
\cline { 2 - 8 } & 0.3 & 5.3165 & 0.0000 & 0.0920 & 0.0920 & -0.4502 & 1.0890 \\
\cline { 2 - 7 } & 0.5 & 5.1264 & 0.0010 & 0.0880 & 0.0890 & -0.3977 & 1.1430 \\
\hline
\end{tabular}




\section{AL-HAJ EBRAHEM \& AL-MASRI}

Table 7: Simulation Results of the Parameter $\beta_{1}$ when $\mathrm{n}=40$

\begin{tabular}{|c|c|c|c|c|c|c|c|}
\hline $\mathrm{X}_{2}$ & $\mathrm{X}_{1}$ & $\mathrm{~W}$ & $\mathrm{~L}$ & $\mathrm{U}$ & $\mathrm{T}$ & Bias & MSE \\
\hline \multirow{4}{*}{0.9} & 0.1 & 5.4639 & 0.0035 & 0.0860 & 0.0895 & -0.4575 & 2.0540 \\
\cline { 2 - 8 } & 0.2 & 4.0934 & 0.0050 & 0.0585 & 0.0635 & -0.2193 & 1.2430 \\
\cline { 2 - 8 } & 0.3 & 4.0963 & 0.0080 & 0.0590 & 0.0670 & -0.2089 & 1.2220 \\
\cline { 2 - 8 } & 0.5 & 5.2896 & 0.0110 & 0.0435 & 0.0545 & -0.2028 & 1.9090 \\
\hline \multirow{4}{*}{1.0} & 0.1 & 5.6772 & 0.0030 & 0.0920 & 0.0950 & -0.4462 & 1.8450 \\
\cline { 2 - 8 } & 0.2 & 3.8432 & 0.0045 & 0.0620 & 0.0665 & -0.2485 & 1.0380 \\
\cline { 2 - 8 } & 0.3 & 3.6264 & 0.0045 & 0.0545 & 0.0590 & -0.1817 & 0.9110 \\
\cline { 2 - 8 } & 0.5 & 4.3539 & 0.0120 & 0.0485 & 0.0605 & -0.1580 & 1.2890 \\
\hline \multirow{4}{*}{1.2} & 0.1 & 4.8035 & 0.0020 & 0.0875 & 0.0895 & -0.4210 & 1.4880 \\
\cline { 2 - 8 } & 0.2 & 3.3155 & 0.0065 & 0.0595 & 0.0660 & -0.1993 & 0.8150 \\
\cline { 2 - 8 } & 0.3 & 3.0358 & 0.0055 & 0.0600 & 0.0655 & -0.1688 & 0.6660 \\
\cline { 2 - 8 } & 0.5 & 3.2786 & 0.0105 & 0.0505 & 0.0610 & -0.1244 & 0.7370 \\
\hline & 0.1 & 4.4699 & 0.0020 & 0.0700 & 0.0720 & -0.3210 & 1.1760 \\
\cline { 2 - 8 } & 0.2 & 3.2118 & 0.0030 & 0.0710 & 0.0740 & -0.2396 & 0.7610 \\
\cline { 2 - 8 } & 0.3 & 2.8369 & 0.0060 & 0.0595 & 0.0655 & -0.1685 & 0.5970 \\
\cline { 2 - 8 } & 0.5 & 2.9173 & 0.0105 & 0.0490 & 0.0595 & -0.1386 & 0.5930 \\
\hline \multirow{4}{*}{1.9} & 0.1 & 3.6709 & 0.0010 & 0.0745 & 0.0755 & -0.2878 & 0.7030 \\
\cline { 2 - 8 } & 0.2 & 2.9251 & 0.0020 & 0.0625 & 0.0645 & -0.1974 & 0.4530 \\
\cline { 2 - 8 } & 0.3 & 2.3678 & 0.0050 & 0.0745 & 0.0795 & -0.1508 & 0.3530 \\
\cline { 2 - 8 } & 0.5 & 1.9082 & 0.0055 & 0.0580 & 0.0635 & -0.1121 & 0.2710 \\
\hline
\end{tabular}

Table 8: Simulation Results of the Parameter $\beta_{1}$ when $\mathrm{n}=80$

\begin{tabular}{|c|c|c|c|c|c|c|c|}
\hline $\mathrm{X}_{2}$ & $\mathrm{X}_{1}$ & $\mathrm{~W}$ & $\mathrm{~L}$ & $\mathrm{U}$ & $\mathrm{T}$ & Bias & MSE \\
\hline \multirow{4}{*}{0.9} & 0.1 & 3.3864 & 0.0045 & 0.0555 & 0.0600 & -0.1634 & 0.8710 \\
\cline { 2 - 8 } & 0.2 & 2.8042 & 0.0110 & 0.0420 & 0.0530 & -0.1308 & 0.5250 \\
\cline { 2 - 8 } & 0.3 & 2.8413 & 0.0140 & 0.0480 & 0.0620 & -0.1052 & 0.5510 \\
\cline { 2 - 8 } & 0.5 & 3.6913 & 0.0115 & 0.0470 & 0.0585 & -0.1321 & 0.9460 \\
\hline \multirow{4}{*}{1.0} & 0.1 & 3.1606 & 0.0025 & 0.0580 & 0.0605 & -0.1898 & 0.7530 \\
\cline { 2 - 8 } & 0.2 & 2.5651 & 0.0080 & 0.0480 & 0.0560 & -0.1370 & 0.4590 \\
\cline { 2 - 8 } & 0.3 & 2.5175 & 0.0125 & 0.0450 & 0.0575 & -0.0806 & 0.4390 \\
\cline { 2 - 8 } & 0.5 & 3.0276 & 0.0125 & 0.0415 & 0.0540 & -0.1065 & 0.6020 \\
\hline \multirow{4}{*}{1.2} & 0.1 & 2.8929 & 0.0080 & 0.0670 & 0.0750 & -0.1713 & 0.6230 \\
\cline { 2 - 8 } & 0.2 & 2.2143 & 0.0090 & 0.0595 & 0.0685 & -0.1175 & 0.3520 \\
\cline { 2 - 8 } & 0.3 & 2.0923 & 0.0110 & 0.0460 & 0.0570 & -0.0861 & 0.2960 \\
\cline { 2 - 8 } & 0.5 & 2.2683 & 0.0115 & 0.0325 & 0.0440 & -0.0689 & 0.3200 \\
\hline \multirow{4}{*}{1.3} & 0.1 & 2.8233 & 0.0035 & 0.0675 & 0.0710 & -0.1744 & 0.5990 \\
\cline { 2 - 8 } & 0.2 & 2.0904 & 0.0070 & 0.0550 & 0.0620 & -0.1297 & 0.3180 \\
\cline { 2 - 8 } & 0.3 & 1.9392 & 0.0160 & 0.0475 & 0.0635 & -0.0719 & 0.2680 \\
\hline \multirow{4}{*}{1.9} & 0.5 & 2.0396 & 0.0140 & 0.0520 & 0.0660 & -0.0658 & 0.2840 \\
\cline { 2 - 8 } & 0.1 & 2.3871 & 0.0030 & 0.0765 & 0.0795 & -0.1722 & 0.4090 \\
\cline { 2 - 8 } & 0.2 & 1.6190 & 0.0065 & 0.0720 & 0.0785 & -0.1069 & 0.2040 \\
\cline { 2 - 8 } & 0.3 & 1.4227 & 0.0085 & 0.0435 & 0.0520 & -0.0585 & 0.1350 \\
\hline & 0.5 & 1.3256 & 0.0120 & 0.0350 & 0.0470 & -0.0468 & 0.1130 \\
\hline
\end{tabular}




\section{RAYLEIGH MODEL PARAMETER ESTIMATION IN STEP-STRESS TESTING}

Table 9: Simulation Results of the Parameter $\beta_{1}$ when $\mathrm{n}=100$

\begin{tabular}{|c|c|c|c|c|c|c|c|}
\hline $\mathrm{X}_{2}$ & $\mathrm{X}_{1}$ & $\mathrm{~W}$ & $\mathrm{~L}$ & $\mathrm{U}$ & $\mathrm{T}$ & Bias & MSE \\
\hline \multirow{4}{*}{0.9} & 0.1 & 2.9543 & 0.0100 & 0.0475 & 0.0575 & -0.1281 & 0.6250 \\
\cline { 2 - 8 } & 0.2 & 2.4986 & 0.0140 & 0.0440 & 0.0580 & -0.0878 & 0.4090 \\
\cline { 2 - 8 } & 0.3 & 2.5221 & 0.0140 & 0.0530 & 0.0670 & -0.0830 & 0.4470 \\
\cline { 2 - 8 } & 0.5 & 3.2879 & 0.0120 & 0.0315 & 0.0435 & -0.0776 & 0.6800 \\
\hline \multirow{4}{*}{1.0} & 0.1 & 2.7718 & 0.0050 & 0.0505 & 0.0555 & -0.1569 & 0.5480 \\
\cline { 2 - 8 } & 0.2 & 2.2803 & 0.0120 & 0.0450 & 0.0570 & -0.0832 & 0.3500 \\
\cline { 2 - 8 } & 0.3 & 2.2438 & 0.0120 & 0.0430 & 0.0550 & -0.0898 & 0.3420 \\
\cline { 2 - 8 } & 0.5 & 2.7064 & 0.0180 & 0.0345 & 0.0525 & -0.0587 & 0.4950 \\
\hline \multirow{4}{*}{1.2} & 0.1 & 2.5031 & 0.0070 & 0.0540 & 0.0610 & -0.1534 & 0.4590 \\
\cline { 2 - 8 } & 0.2 & 1.9714 & 0.0080 & 0.0480 & 0.0560 & -0.0793 & 0.2750 \\
\cline { 2 - 8 } & 0.3 & 1.8585 & 0.0115 & 0.0465 & 0.0580 & -0.0556 & 0.2260 \\
\cline { 2 - 8 } & 0.5 & 2.0282 & 0.0125 & 0.0385 & 0.0510 & -0.0463 & 0.2790 \\
\hline \multirow{4}{*}{1.3} & 0.1 & 2.4068 & 0.0035 & 0.0670 & 0.0705 & -0.1662 & 0.4800 \\
\cline { 2 - 8 } & 0.2 & 1.8635 & 0.0105 & 0.0395 & 0.0500 & -0.0847 & 0.2290 \\
\cline { 2 - 8 } & 0.3 & 1.7225 & 0.0090 & 0.0440 & 0.0530 & -0.0654 & 0.1960 \\
\cline { 2 - 8 } & 0.5 & 1.8186 & 0.0190 & 0.0355 & 0.0545 & -0.0412 & 0.2250 \\
\hline \multirow{4}{*}{1.9} & 0.1 & 1.9923 & 0.0035 & 0.0735 & 0.0770 & -0.1514 & 0.3130 \\
\cline { 2 - 8 } & 0.2 & 1.4353 & 0.0075 & 0.0575 & 0.0650 & -0.0729 & 0.1530 \\
\cline { 2 - 8 } & 0.3 & 1.2630 & 0.0085 & 0.0430 & 0.0515 & -0.0534 & 0.1110 \\
\cline { 2 - 7 } & 0.5 & 1.1779 & 0.0115 & 0.0405 & 0.0520 & -0.0352 & 0.0920 \\
\hline
\end{tabular}

References

Al-Haj Ebrahem, M., \& Al Masri, A. (2007(a)). Optimum simple step-stress plan for log-logistic cumulative exposure model. Metron Iternational Journal of Statistics, LXV(1), 2334.

Al-Haj Ebrahem, M., \& Al Masri, A. (2007(b)). Optimum simple step-stress plan for log-logistic distribution under time censoring. Abhath Al-Yarmouk: Basic Sci. and Eng., 16, 319-327.

Alhadeed, A., \& Yang, S. (2005). Optimal simple step-stress plan for cumulative exposure model using log-normal distribution. IEEE Transactions on Reliability, 54, 64-68.

Bai, D., Kim, M., \& Lee, S. (1989). Optimum simple step-stress accelerated life tests with censoring. IEEE Transactions on Reliability, 38, 528-532.

Chung, S., \& Bai, D. (1998). Optimal designs of simple step-stress accelerated life tests for lognormal lifetime distributions. International Journal of Reliability, Quality and Safety Engineering, 5, 315-336.
Cohen, A., \& Whitten, B. (1988). Parameter estimation in reliability and life span models. New York, Marcel Dekker.

Gouno, E. (2001). An inference method for temperature step-stress accelerated life testing. Quality and Reliability Engineering International, 17, 11-18.

Johnson, N., Kotz, S., \& Balakrishnan, N. (1994). Continuous univariate distributions: Volume 1. New York: Wiley.

Khamis, I., \& Higgins, J. (1996). Optimum 3-step step-stress tests. IEEE Transactions on Reliability, 45, 341-345.

Khamis, I., \& Higgins, J. (1996). An alternative to the Weibull step-stress model. Proceedings of the American Statistical Association, Chicago, 123-127.

Khamis, I., \& Higgins, J. (1998). A new model for step-stress testing. IEEE Transactions on Reliability, 47, 131-134.

Miller, R., \& Nelson, B. (1983). Optimum simple step-stress plans for accelerated life testing. IEEE Transactions on Reliability, 32, 59-65. 


\section{AL-HAJ EBRAHEM \& AL-MASRI}

Nelson, W. (1980). Accelerated life testing step-stress models and data analysis. IEEE Transactions on Reliability, 29, 103-108.

Nelson, W. (1990). Accelerated testing, statistical models, test plans, and data analysis. New York: Wiley.

Schmoyer, R. (1991). Nonparametric analysis for two-level single-stress accelerated life tests. Technometrics, 33, 175-186.

Shaked, M., \& Singurwalla, N. (1983). Inference for step-stress accelerated life tests. Journal of Statistical Planning and Inference, 7, 295-306.
Xiong, C. (1998). Inferences on a simple step-stress model with type-II censored exponential data. IEEE Transactions on Reliability, 47, 142-146.

Xiong, C., \& Milliken, G. (2002). Prediction for exponential lifetimes based on step-stress testing. Communications in StatisticsSimulation, 31, 539-556. 